Medicina Experimentalis 1962;7(suppl 1):193-195

\title{
Namen und Anschriften der deutschen Teilnehmer
}

Liste der deutschen Teilnehmer

Dr. H. Bayreuther Dr. D. Bente

Psychiatrische und Nervenklinik der Universität Marburg/Lahn Oberarzt der Nervenklinik der Universität Erlangen

Priv.-Doz. Dr. W. Bredemann

Oberarzt der Psychiatrischen und Neurologischen Klinik der Freien Universität Berlin, BerlinCharlottenburg (jetzt: Chefarzt der Landes-Nervenklinik, Berlin-Spandau)

Dr. W. Bruscha Prof. Dr. H. Büssow

Nervenklinik der Universität Erlangen

Direktor des Allgem. Krankenhauses Hamburg-Ochsenzoll, Langenhorner Chaussee 560

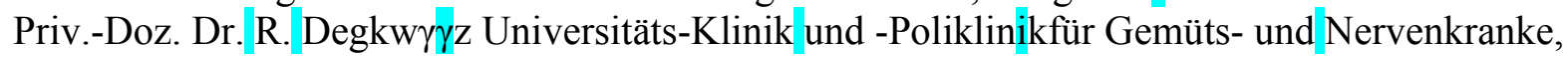
Frankfurt/ Main

Prof. Dr. W. Doehner

Prof. Dr. W. Ederle Prof. Dr. W. Eicke

Psychiatrische und Neurologische Klinik der Universität Kiel (jetzt: Direktor des Psychiatrischen Landeskrankenhauses Schleswig)

Direktor der Heilanstalt Weißenau bei Ravensburg

Direktor des Psychiatrischen Landeskrankenhauses Marburg/Lahn

Priv.-Doz. Dr. M.-P. Engelmeier

Psychiatrische und Nervenklinik der Universität Münster/Westf.

Prof. Dr. F. Flügel

Dr. W. Fuchs

Frau Dr. E. B. Gäde

Direktor der Nervenklinik der Universität Erlangen Oberarzt der Nervenklinik der Städt.

Krankenanstalten Essen Mainz, Thauernstraße 35

Priv.-Doz. Dr. Dr. H.-J. Haase

Psychiatrische Klinik der Med. Akademie und Rhein. Landes-krankenhaus Düsseldorf

Dr. G. Hackstein Dr. K. Hartmann

Priv.-Doz. Dr. H. Heyck

Dr. K. Heinrich Dr. J. Hiob Dr. H. Hippius

Landesheilanstalt Süchteln/Rheinland

Psychiatrische und Neurologische Klinik der Freien Universität Berlin, Berlin-Charlottenburg (jetzt: Leitender Arzt des Jugend-psychiatrischen Beobachtungsheims «Grünes Haus», BerlinTegel)

Oberarzt der Psychiatrischen und Neurologischen Klinik der Freien Universität Berlin, Berlin-

Charlottenburg (jetzt: Berlin-Dahlem, Clayallee 249)

Psychiatrische und Nervenklinik der Universität Mainz

Berlin-Grunewald, Karlsbader Straße 12c 
Psychiatrische und Neurologische Klinik der Freien Universität Berlin, Berlin-Charlottenburg 193

Prof. Dr. J. Hirschmann Dr. T. Itil

Universitäts-Nervenklinik Tubingen Nervenklinik der Universität Erlangen

Priv.-Doz. Dr. W. Janzarik Oberarzt der Psychiatrischen und Nervenklinik der Universität Mainz

Dr. H.-J. Jessel

Dr. K. Kanig

Dr. H. Kemper Priv.-Doz. Dr. R. Klaue

Prof. Dr. H. Kranz

Dr. P. Krauss

Prof. Dr. F. Laubénthal

Dr. L. Leisten

Dr. H. Lieser

Dr. B. MOLDENHAUER

Frau Dr. B. Molitoris Frau Dr. M. Müller

Allgem.KrankenhausHamburg-Ochsenzoll,LangenhornerChaus-see 560

Psychiatrische und Neurologische Klinik der Freien Universität Berlin, Berlin-Charlottenburg

Oberarzt im Oldenburger Landeskrankenhaus Wehnen i. O.

Ärztlicher Direktor der Karl-Bonhoeffer-Heilstätten, Berlin-Wittenau

Direktor der Psychiatrischen und Nervenklinik der Universität Mainz

Chefarzt der Psychiatrischen Klinik Christophsbad, Göppingen

Chefarzt der Nervenklinik der Städt. Krankenanstalten, Essen

Pharm. Wiss. Beratung, Farbenfabriken Bayer Leverkusen

Nervenkrankenhaus Haar b. München

Oberarzt der Karl-Bonhoeffer-Heilstätten, Berlin-Wittenau

Universitäts-Nervenklinik Erlangen

Universitäts-Nervenklinik Erlangen

Dr. D. Müller-Plettenberg

Wuppertal-Barmen, Herzkamperstraße la

Dr. Dr. H. Neumann Dr. Dr. H.-E. Richter

Prof. Dr. W. Scheid

Psychiatrisches Landeskrankenhaus Emmendingen

Psychiatrische und Neurologische Klinik der Freien Universität Berlin, Berlin-Charlottenburg

Direktor der Universitäts-Nervenklinik Köln, Köln-Lindenthal

Priv.-Doz. Dr. W. Schm $\gamma \gamma t$ Oberarzt der Psychiatrischen und Neurologischen Klinik der

Universität des Saarlandes, Homburg/Saar

Dr. V. Schultze Prof. Dr. H. Selbach

Dr. J. Sommer

Dr. G. Steuber Dr. R. Tölle

Universitäts-Nervenklinik Göttingen

Direktor der Psychiatrischen und Neurologischen Klinik der Freien Universität Berlin, Berlin-

Charlottenburg

Pharm. Wiss. Med. Entwicklung, Farbenfabriken Bayer Leverkusen

Direktor des Niedersächsischen Landeskrankenhauses in Lüneburg

Psychiatrische Klinik Christophsbad, Göppingen (jetzt: Universitäts-Nervenklinik Tubingen) 
Prof. Dr. H. H. Wieck

Oberarzt der Nervenklinik der Universität Köln, Köln-Lindenthal 194 